\title{
Phase Transitions of Dimeric Liquid Crystals Containing Long Odd-Numbered Methylene Spacers
}

\author{
Yoshihiro OGaWA ${ }^{\dagger}$ and Kazuya Ootani* \\ Department of Chemistry, Faculty of Science, Kumamoto University, \\ Kurokami, Kumamoto 860-8555, Japan \\ * Department of Applied Science, Faculty of Engineering, Kyushu University, \\ Fukuoka 812-8581, Japan
}

(Received June 18, 1998)

\begin{abstract}
A series of $\alpha, \omega$-bis $\{4-(4-b i p h e n y l o x y c a r b o n y l) p h e n y l\}$ alkanedioates (BPA- $n$ ) dimeric liquid crystals having long odd-numbered methylene spacers was prepared. The number of methylene groups in the flexible alkyl spacer has been varied from 9 to 21 . Long period $L$ of crystalline BPA- $n$ obtained by X-ray powder diffraction were shown as a linear equation of the number $n$ of methylene units, as follows, $L=1.16 n+35.2(\AA)$. BPA-21 showed no liquid crystalline phase. BPA-19 is monotropic nematic. The other BPA- $n$ showed an enantiotropic nematic phase. The overall entropies $\Delta S_{\mathrm{KI}}$ of the transition from the crystals at room temperature to the isotropic liquid were also shown as a linear equation of $n$, as follows, $\Delta S_{\mathrm{KI}}=8.54 n+94.8\left(\mathrm{~J} \mathrm{~K}^{-1} \mathrm{~mol}^{-1}\right)$. The crystal-nematic $\left(T_{\mathrm{KN}}\right)$ transition temperature shows a weak odd-even oscillation, but the nematic-isotropic $\left(T_{\mathrm{NI}}\right)$ transition temperature showed a distinctive odd-even effect. The odd-even effect of a $T_{\mathrm{NI}}$ decreases with an increase in $n$. The nematic-isotropic $\left(\Delta S_{\mathrm{Nl}}\right)$ entropy change is almost constant with an increase in $n$ with a strong odd-even oscillation, higher values being detected for the homologues with even-numbered spacers.

KEY WORDS Thermotropic Liquid Crystalline Polymer / Dimeric Liquid Crystals / Odd-Numbered
\end{abstract} Methylene Spacers / Thermal Properties / Odd-Even Effects /

Main chain liquid crystalline polymers with repeated units of mesogen spacers have a characteristic that the odd-even effect of spacer length is more distinctive than the low molecular liquid crystals. ${ }^{1}$ This odd-even effect is observed for the nematic-isotropic phase transition temperature and associated entropy change. As most of the dimeric liquid crystals formed by a sequence of mesogen-spacer-mesogen showed very similar odd-even effect to the liquid crystalline polymers, they have been extensively studied as models for polymers. ${ }^{2-5}$ The recent interest of this dimers stems from their quite different properties to conventional low molecular liquid cryatals. ${ }^{6,7}$

There is an approach to understand the thermal properties of flexible molecules. It is to separate thermodynamic quantities the contributions of methylene units and terminal groups. For example, thermal properties for $n$-alkanes have been treated on the supposition that both the enthalpy and entropy of melting may be adequately represented by linear expressions, each comprising a term proportional to the number of methylene units and an additive constant for contributions associated with the two methyl groups. ${ }^{8}$ There have been a number of studies of the thermal properties of dimers, but there had been no reports that treats such thermodynamic quantity quantitatively with separation of contribution of mesogens and contribution of polymethylene spacer parts, respectively. In a previous paper, we reported a new series of dimeric liquid crystals with an even-number long methylene spacers from $n=8$ to $n=20$, as shown below ${ }^{9}$ :

\footnotetext{
† To whom correspondence should be addressed.
}

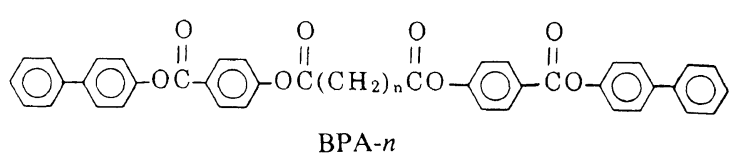

We found that the overall entropies $\Delta S_{\mathrm{KI}}$ transition from crystals at room temperature to the isotropic liquid were shown as a linear equation of the number $n$ of methylene units. The entropies of transition from the nematic to isotropic phase were almost constant, independent of $n$ in the flexible spacer.

The first goal of this paper is to show that the thermodynamic quantities of the dimeric liquid crystals with an odd-numbered long methylene spacers are classified as contributors from mesogens and polymethylene spacers. Such a treatment is valid only during the same crystal structure. Dimers that have the same crystal structure will be obtained only when the spacer has a sufficiently long chain. Therefore, $\alpha, \omega$-bis $\{4-(4-$ biphenyloxycarbonyl)phenyl $\}$ alkanedioates (BPA- $n$ ) with long methylene units from 9 to 21 were used in this study. The second goal of this paper is to summarize and discuss the odd-even effect of BPA- $n$. There have been a number of studies of the odd-even effects for dimers below $n=13$, but there has been no report of $n=15$ or above. We have been interested on how an odd-even effect of dimers converges, when $n$ gets longer. The synthesis and phase behavior of dimers containing a long methylene chain will be described in this paper.

\section{EXPERIMENTAL}

\section{Materials}

Suberic, azelaic, sebacic, undecanedioic, dodecanedioic and tridecanedioic acid (all from Tokyo Kasei) were converted to the methyl esters by the conventional procedures and fractionally distilled to yield materials 
Table I. Elemental analysis of dimers BPA- $n$

\begin{tabular}{lcc}
\hline BPA- $n$ & $\mathrm{C} / \%$ & $\mathrm{H} / \%$ \\
\hline BPA-9 & $77.24(77.35)^{\mathrm{a}}$ & $5.85(5.83)$ \\
BPA-11 & $77.84(77.64)$ & $6.11(6.13)$ \\
BPA-13 & $77.65(77.92)$ & $6.43(6.42)$ \\
BPA-15 & $78.07(78.17)$ & $6.72(6.68)$ \\
BPA-17 & $78.30(78.41)$ & $6.96(6.93)$ \\
BPA-19 & $78.75(78.63)$ & $7.16(7.16)$ \\
BPA-21 & $79.01(78.85)$ & $7.41(7.38)$ \\
\hline
\end{tabular}

${ }^{a}$ Values in parentheses are those calculated.

which analyzed $99.8 \%$ by capillary gas chromatography. $p$-Hydroxybenzoic acid and $p$-phenylphenol (all from Tokyo Kasei) were used as received.

\section{Long Chain $\alpha, \omega$-Dicarboxylic Acids}

Pentadecanedioic, heptadecanedioic, nonadecanedioic, heneicosanedioic, and tricosanedioic acid were prepared from diacids by use of the ketene dimerization synthesis. ${ }^{10}$ For example, pentadecanedioic acid was prepared by the Wolff-Kishner reduction of 8-ketopentadecanedioic acid, formed by dimerization of acid chloride of monomethyl hydrogen octanedioate. Dimethyl esters of the diacids synthesized were purified by fractional distillation and their purity were $99.8 \%$ by capillary gas chromatography.

\section{Synthesis of $B P A-n$}

The synthesis of BPA- $n$ has been previously described. ${ }^{9}$ The results of elemental analysis have been found to be in good agreement with the calculated values shown in Table I. The samples were obtained by slow crystallization from toluene solution.

\section{Measurements}

Transition temperatures and enthalpies of BPA- $n$ were measured on a Rigaku DSC 8240 differential scanning calorimeter at a heating rate of $3 \mathrm{Kmin}^{-1}$. Heat quantity was taken from the peak area of DSC based on indium as the standard. Optical textures were determined using an Olympas $\mathrm{BH}-2$ optical polarizing microscope with heating stage Mettler FP80/82.

\section{RESULTS AND DISCUSSION}

\section{Long Periods}

X-Ray long periods offer a fairly convenient check on the structure of a given BPA- $n$. Figure 1 shows the plots of long period $L$ of crystalline BPA- $n$ obtained by X-ray powder diffraction versus the number $n$ of methylene units. For an odd number series, a linear equation is,

$$
L=1.16 n+35.2(\AA)
$$

The crystal structure is not determined, but it is thought that odd number series BPA- $n$ has the same crystal structure because of the linearity of $L$ versus $n$. The crystal structure of $\alpha, \omega$-bis(4-cyanobiphenyl-4'oxy)heptane was determined from single crystal X-ray diffractometry and it was shown that the flexible spacer was in the all trans-conformation. ${ }^{11}$ Similarly a polymethylene spacer in a molecule for BPA- $n$ is assumed to form an extending zigzag structure which has the length $1.27 \AA$ per 52

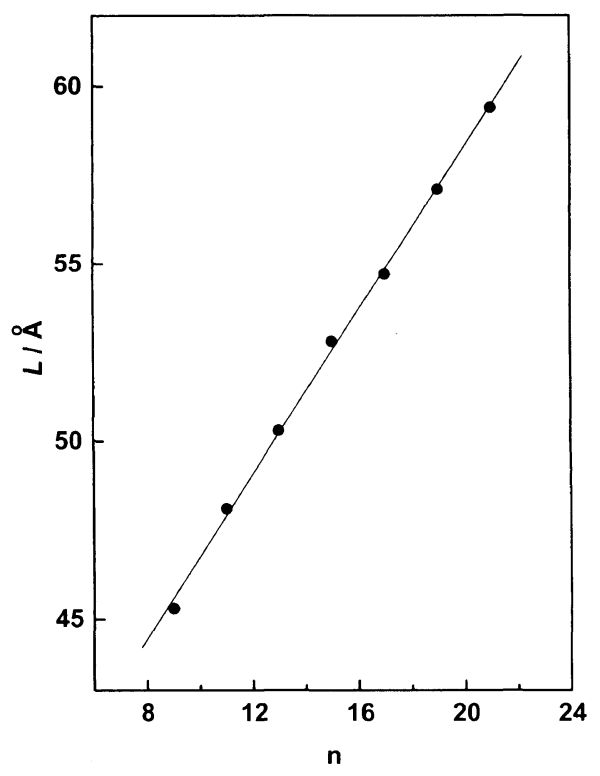

Figure 1. Long period $L$ of crystalline BPA- $n$ versus the number $n$ of methylene units in a flexible spacer.

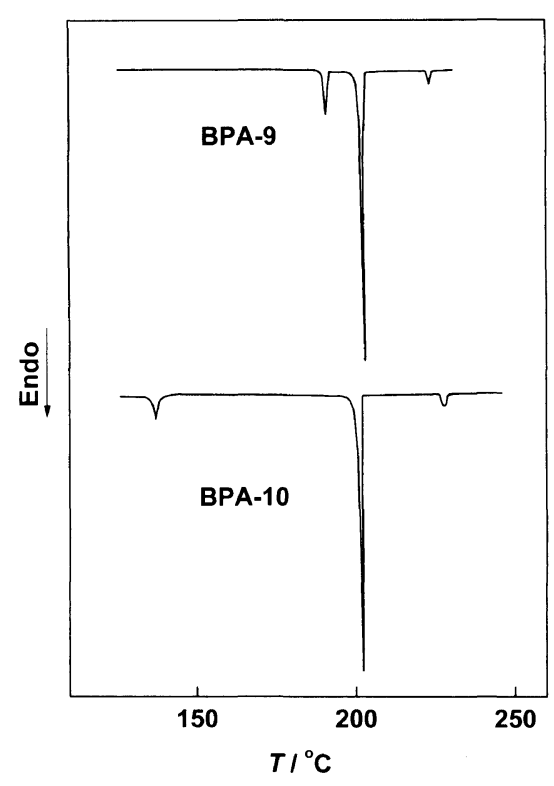

Figure 2. DSC heating curves of BPA-9 and BPA-10.

methylene. The angle between the polymethylene chain and the layer was thus obtained by the equation $\sin \theta=$ $1.16 / 1.27$, and becomes $66^{\circ}$. The value $35.2 \AA$ of the second term in formula (1) is a little longer than the calculated value $33.8 \AA$ of length of two mesogens. It is thought that well ordered dimer crystal adjacent lamellae are separated by a plane layer of void and its thickness is $1.4 \AA$.

\section{Thermal Properties}

Figure 2 shows DSC heating curves of BPA-9 and BPA-10. On heating, three endothermic peaks were found respectively. The sequence of phase transitions is as follows: solid-solid, solid-nematic, nematic-isotropic, because Schlieren texture which is characteristic of nematic was observed after melting by polarizing microscope. A solid-solid transition temperature and a melting temperature of BPA-9 are higher than those of 
Table II. Long periods $L$ and thermodynamic properties of crystalline BPA- $n$

\begin{tabular}{|c|c|c|c|c|c|c|}
\hline \multirow{2}{*}{$\mathrm{BPA}-n$} & $L$ & $T_{\mathrm{KN}}$ & $T_{\mathrm{NI}}$ & $\Delta S_{\mathrm{K} 1}$ & $\Delta S_{\mathrm{NI}}$ & \multirow{2}{*}{$\Delta S_{\mathrm{NI}} / \Delta S_{\mathrm{Kl}}$} \\
\hline & $\AA$ & ${ }^{\circ} \mathrm{C}$ & ${ }^{\circ} \mathrm{C}$ & $\mathrm{J} \mathrm{K}^{-1} \mathrm{~mol}^{-1}$ & $\mathrm{~J} \mathrm{~K}^{-1} \mathrm{~mol}^{-1}$ & \\
\hline BPA-9 & 45.3 & 202 & 224 & 171 & 7.0 & 0.041 \\
\hline BPA-11 & 48.1 & 198 & 216 & 192 & 7.2 & 0.037 \\
\hline BPA-13 & 50.3 & 194 & 207 & 204 & 7.1 & 0.035 \\
\hline BPA-15 & 52.8 & 191 & 198 & 219 & 7.2 & 0.033 \\
\hline BPA-17 & 54.7 & 188 & 191 & 243 & 7.0 & 0.029 \\
\hline BPA-19 & 57.1 & $185^{\mathrm{a}}$ & - & 257 & - & - \\
\hline BPA-21 & 59.4 & $182^{\mathrm{a}}$ & - & 274 & - & - \\
\hline
\end{tabular}

${ }^{\mathrm{a}} T_{\mathrm{KI}}$.

BPA-10 and, whereas a nematic-isotropic transition temperature of BPA-9 is lower than that of BPA-10.

BPA-21 showed no liquid crystalline phase. The other synthesized odd-numbered dimers displayed nematic mesophase which was enantiotropic for $n=8-17$ and monotropic for $n=19$. Thermodynamic data for each dimer are given in Table II. Including solid-solid transition, the overall entropy change $\Delta S_{\mathrm{KI}}$ of transition from crystals at room temperature to the isotropic liquid is plotted as a function of $n$ as shown in Figure 3 . Obtained linear function is expressed as

$$
\Delta S_{\mathrm{KI}}=8.54 n+94.8\left(\mathrm{~J} \mathrm{~K}^{-1} \mathrm{~mol}^{-1}\right)
$$

This equation demonstrates the additivity of the contribution from the polymethylene spacers and mesogens. Therefore, eq 2 can be rewritten as: $\Delta S_{\mathrm{KI}}=\Delta S_{\mathrm{KI}}^{\mathrm{F}} n+$ $\Delta S_{\mathrm{KI}}^{\mathrm{M}} \cdot \Delta S_{\mathrm{KI}}^{\mathrm{F}}$ is the entropy change of the methylene unit in the flexible spacer, and $\Delta S_{\mathrm{KI}}^{\mathrm{M}}$ is the entropy change of the mesogens, at the crystal-isotropic transition. The $\Delta S_{\mathrm{KI}}^{\mathrm{F}}$ and $\Delta S_{\mathrm{KI}}^{\mathrm{M}}$ of dimers having odd-numbered methylene spacers were 8.54 and $94.8\left(\mathrm{~J} \mathrm{~K}^{-1} \mathrm{~mol}^{-1}\right)$ respectively and those values are almost the same to the values of even-numbered dimers, 8.50 and 95.4. ${ }^{9}$ There is no odd-even effect for the overall entropy change at crystal-isotropic transition.

For liquid crystalline polymers, $\Delta S_{\mathrm{NI}} / \Delta S_{\mathrm{KI}}$ is often used as a measure to represent the degree of order in the nematic phase relative to that of the crystal phase. Here, $\Delta S_{\mathrm{NI}}$ is the entropy change of nematic-isotropic (N-I) phase transition and $\Delta S_{\mathrm{KI}}$ is the entropy change of crystal-isotropic (K-I) phase transition. These values for BPA- $n$ were $0.03-0.04$, as shown in Table II, similar those of RF-type(rigid-flexible sequence) and F-R-F type (flexible-rigid-flexible) low molecular liquid crystals which is $0.02-0.03,{ }^{12}$ and are considerably smaller than those of oligomers $0.1-0.2^{13}$ and polymer $0.1-0.3^{14}$. Therefore, the nematic regularity of dimers having a odd-numbered spacer is almost the same as that of low molecular liquid crystals.

\section{Odd-Even Effects}

Figure 4 shows the crystal-nematic $\left(T_{\mathrm{KN}}\right)$ and nematic-isotropic $\left(T_{\mathrm{NI}}\right)$ transition temperatures versus spacer length. The data of even-numbered dimers are reported in previous paper. ${ }^{9}$ Both transition temperatures, $T_{\mathrm{KN}}$, and $T_{\mathrm{Nl}}$, were found to decrease with an increase in $n$. $T_{\mathrm{KN}}$ shows slightly odd-even effect, but $T_{\mathrm{NI}}$ shows a distinctive odd-even effect. The odd-even effect of the $T_{\mathrm{NI}}$ decreases with an increase in $n$. This trend

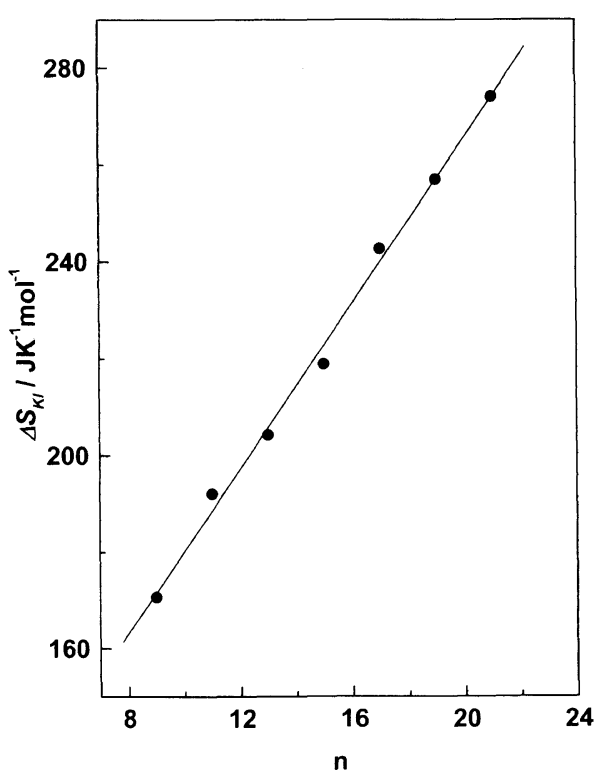

Figure 3. Overall entropy change $\Delta S_{\mathrm{KI}}$ of transition from crystals at room temperature to the isotropic liquid as a function of $n$.

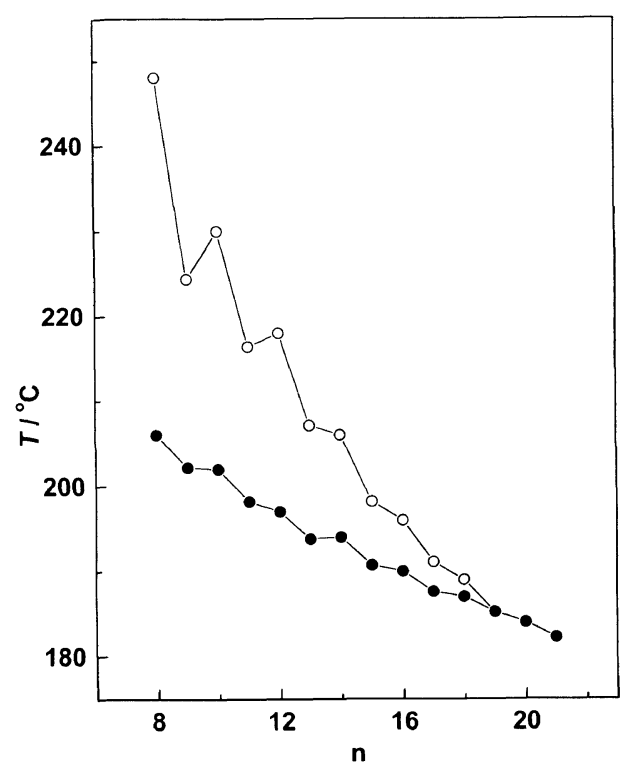

Figure 4. Dependence of crystal-nematic, $T_{\mathrm{KN}}(\bigcirc)$, and nematic-isotropic, $T_{\mathrm{NI}}(\bigcirc)$, transition temperatures versus $n$.

provides a vanishing of the odd-even dependence for the long flexible spacers. As $T_{\mathrm{NI}}$ more closely depends on $n$ than $T_{\mathrm{KN}}$, the temperature range over which the 


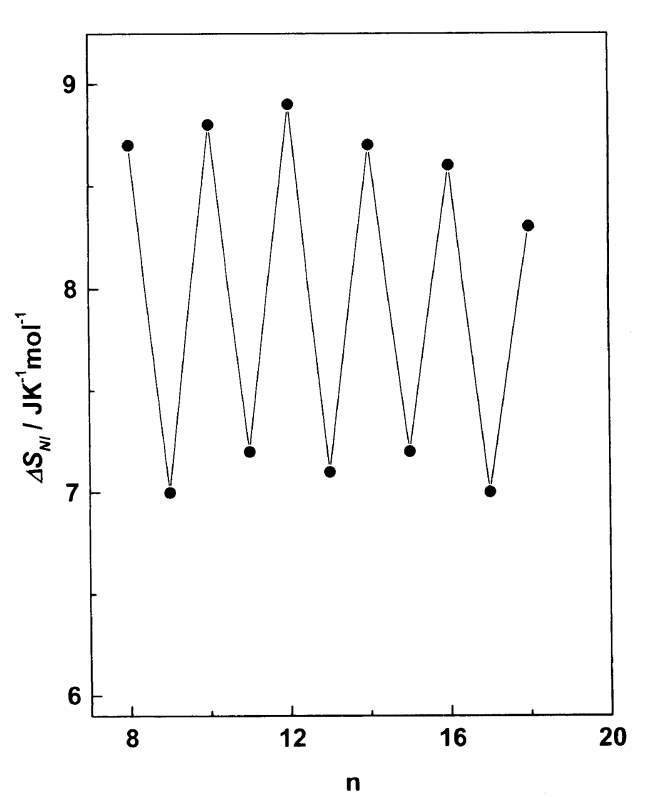

Figure 5. Dependence of nematic-isotropic transition entropy change, $\Delta S_{\mathrm{NI}}$, versus $n$.

nematic phase exists becomes smaller with an increase in the spacer length, and finally at $n=19$ it no longer shows liquid crystals during heating. By use of the rotational-isomeric-state approximation, this odd-even effect has been explained by the different orderings of the mesogens in the nematic phase depending on the number of flexible units in the spacer. ${ }^{15}$ This conclusion has been well confirmed by the experimental results obtained by ${ }^{2} \mathrm{H}$ NMR measurement. ${ }^{16,17}$

Figure 5 plots the entropy changes associated with the nematic-isotropic $\left(\Delta S_{\mathrm{NI}}\right)$ phase transition as a function of $n . \Delta S_{\mathrm{NI}}$ showed a marked odd-even oscillation and continue to be almost constant for odd-numbered and even-numbered BPA- $n$, respectively, higher values being detected for the homologues with even-numbered spacers. This odd-even effect of $\Delta S_{\mathrm{NI}}$ is observed even just before nematic phase disappears at $n=19$. The value of even dimers is approximately 20 percent more than that of odd dimers. The magnitude to the odd-even effects for these dimers seems to be somewhat smaller than that for other dimers or polymers. It can be understood by the difference of the nature of the link joining the spacer to the mesogen groups. ${ }^{18,19}$ It is said that a better ordered nematic phase is obtained with an even number of flexible units in the spacer, leading to a higher nematic-isotropic phase transition temperature and a higher associated entropy change. The odd-even effect of $T_{\mathrm{NI}}$ is attenuated with increase of $n$. In contrast, that of $\Delta S_{\mathrm{NI}}$ is essentially unattenuated with increase in $n$. Ferrarini et al. predicted the existence of a nematic-nematic transition for liquid crystal dimers. ${ }^{20}$ Shimizu et al. also reported that the ordering of the mesogens and of the molecule itself to increase with a decrease in temperature in the nematic phase, whereas that of spacer remains almost constant. ${ }^{21}$ It is thought that the difference in the $n$ dependency of $T_{\mathrm{NI}}$ and $\Delta S_{\mathrm{NI}}$ results from an alteration of the ordering of mesogens in the nematic phase. This point will be clarified based on additional study on $n$ dependence of $T_{\mathrm{NI}}$ and $\Delta S_{\mathrm{NI}}$ for many dimeric liquid crystals which have various mesogens.

Acknowledgments. We would like to thank the late Professor K.Takamizawa, Kyushu University, for his helpful discussions.

\section{REFERENCES}

1. A. Griffin and T. Britt, J. Am. Chem. Soc., 103, 4957 (1981).

2. R. B. Blumstein and E. Stickles, Mol. Cryst, Liq. Cryst., 82, 151 (1982).

3. G. R. Luckhurst, Macromol. Symp., 96, 1 (1995).

4. A. Abe and S. Y. Nam, Macromolecules, 28, 90 (1995).

5. A. Abe, H. Furuya, R. N. Shimizu, and S. Y. Nam, Macromolecules, 28, 96 (1995).

6. R. Date, C. Imrie, G. R. Luckhurst, and J. Seddon, Liquid Crystals, 12, 203 (1992).

7. H. Ishizuka, I. Nishiyama, and A. Yoshizawa, Liquid Crystals, 18, 775 (1995).

8. M. G. Broadhurst, J. Res. Nat. Bur. Std., 67A, 233 (1963).

9. Y. Ogawa, Polym. J., 28, 326 (1996).

10. A. Blomquist, J. Johnson, L. Diuguid, J. Shillington, and R. Spencer, J. Am. Chem. Soc., 74, 4203 (1952).

11. L. Malpezzi, S. Brückner, E. Galbiati, and G. R. Luckhurst, Mol. Cryst. Liq. Cryst., 195, 179 (1991).

12. G. W. Gray, "Molecular Structure and the Properties of Liquid Crystals," Academic Press, New York, N.Y., 1962, p 58.

13. Y. Ogawa, Polym. J., 28, 289 (1996).

14. V. Percec and M. Kawasumi, Mcromolecules, 24, 6318 (1991).

15. A. Abe, Macromolecules, 17, 2280 (1984).

16. H. Toriumi, H. Furuya, and A. Abe, Polym. J., 17, 895 (1985).

17. H. Furuya, K. Asahi, and A. Abe, Polym. J., 18, 779 (1986).

18. A. Abe and H. Furuya, Kobunshi Ronbunshu, 43, 247 (1986).

19. G. R. Luckhurst, Macromol. Symp., 96, 1 (1995).

20. A. Ferrarini, G. R. Luckhurst, P. L. Nordio, and S. J. Roskilly, Chem. Phys, Lett., 214, 409 (1993).

21. R. N. Shimizu, H. Kurosu, I. Ando, A. Abe, H. Furuya, and S. Kuroki, Polym. J., 29, 598 (1997). 